Journal of

\section{Applied Crystallography}

ISSN 0021-8898

Received 16 August 2006

Accepted 27 February 2007

\title{
Study of ferrite ferrofluids by small-angle scattering of polarized neutrons
}

\author{
Massimo Bonini, ${ }^{a}$ Albrecht Wiedenmann ${ }^{\mathrm{b}}$ and Piero Baglioni ${ }^{\mathrm{a} *}$ \\ aDepartment of Chemistry and CSGI, University of Florence, via della Lastruccia 3, 50019 Firenze, Italy, and

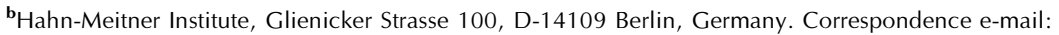 \\ baglioni@csgi.unifi.it
}

\begin{abstract}
Nanoparticles consisting of a magnetic core $\left(\mathrm{Fe}_{3} \mathrm{O}_{4}, \mathrm{CoFe}_{2} \mathrm{O}_{4}\right.$ and $\left.\mathrm{CuFe}_{2} \mathrm{O}_{4}\right)$ and a hydrophobic shell were prepared by chemical co-precipitation of the inorganic cores and by subsequently modifying the surface with dodecanoic acid. The nanoparticles were then dispersed in cyclohexane to form stable ferrofluids. These dispersions were investigated by small-angle scattering of polarized neutrons and the data were interpreted according to a 'pearl-necklace' model, opportunely modified to account for the core-shell structure of the particles. Results of the fitting show that the particles consist of a magnetic core with a mean radius of $40-50 \AA$ and an organic shell with a thickness of $7-8 \AA$. These nanoparticles assemble in fractal aggregates when a magnetic field is applied.
\end{abstract}

(C) 2007 International Union of Crystallography Printed in Singapore - all rights reserved
Small-angle scattering is a technique well suited for the characterization of complex colloidal fluids (Guinier \& Fournet, 1955). In particular, the size and size distribution of suspensions of nanoparticles can be obtained from the intensity of small-angle neutron scattering patterns. It has been shown that when using polarized neutrons the relative contrasts for small-angle scattering of magnetic systems are strongly modified (Keller et al., 2000; Wiedenmann, 2000, 2002; Wiedenmann et al., 2002). In this paper, we used this property to study magnetic fluids obtained from the dispersion in cyclohexane of hydrophobically modified ferrite nanoparticles. The magnetic nanoparticles were obtained through the co-precipitation of the precursors in alkaline conditions and by subsequently grafting a fatty acid onto the surface of the particles. The analysis of the scattering data allowed a complete description of the size, size distribution and layer thickness of the core-shell ferrite nanoparticles. Moreover, the results of the fitting provided important information about the formation of aggregates under an external magnetic field. It is well known that some ferrofluids form aggregates under an applied magnetic field through the alignment of the magnetic dipoles along the direction of the field, while others do not (Jacobs \& Bean, 1963; de Gennes \& Pincus, 1970; Liu et al., 1995). Several experimental and theoretical studies have been performed in order to explain the formation of these aggregates; nevertheless, this process is still not completely understood (Toor, 1993; Gross \& Kiskamp, 1997; Lalatonne et al., 2004). Here we report an analysis based on the so-called 'pearlnecklace' model, originally applied to protein micellized surfactant systems.

\section{Experimental}

\subsection{Reagents}

Iron(II) chloride tetrahydrate (99\%), iron(III) chloride hexahydrate $(97 \%)$, tetramethylammonium hydroxide $(25 \%$ solution in water) and deuterated cyclohexane (99.9\%) were purchased from Aldrich. Copper(II) chloride dihydrate (99\%), sodium hydroxide 
(99\%), cobalt(II) nitrate hexahydrate (99\%), concentrated nitric acid (90\%), iron(III) nitrate nonahydrate $(>98 \%)$, dodecanoic acid (99\%), methyl alcohol (99.5\%) and acetone (99.5\%) were purchased from Fluka. Cobalt(II) chloride hexahydrate (99\%) was purchased from Carlo Erba. All the reagents were used as received.

\subsection{Synthesis of magnetic nanoparticles}

Ferrofluids were synthesized using minor modifications of Massart's method (Massart, 1980, 1981). Aqueous solutions with $1 M$ concentration of the $\mathrm{M}^{2+}$ and $\mathrm{Fe}^{3+}$ cations (where $\mathrm{M}^{2+}$ is $\mathrm{Fe}^{2+}, \mathrm{Co}^{2+}$ or $\mathrm{Cu}^{2+}$ ) were prepared by dissolving the corresponding chloride salts in deoxygenated water (nitrogen bubbling for $0.5 \mathrm{~h}$ before preparation of the solutions). Solutions containing $\mathrm{Fe}^{3+}(10 \mathrm{ml})$ and $M^{2+}(5 \mathrm{ml})$ were mixed and added to concentrated nitric acid $(0.5 \mathrm{ml})$. At this stage, the $\mathrm{M}^{2+} / \mathrm{Fe}^{3+}$ mixture is kept in an acidic medium in order to avoid possible metal hydrolysis. This mixture and $65 \mathrm{ml}$ of $1 M$ sodium hydroxide aqueous solution were separately heated up to boiling point and then mixed as fast as possible under vigorous stirring. The boiling temperature and the stirring were maintained for $2 \mathrm{~h}$. The precipitate was then collected by magnetic decantation, washed twice with water and dispersed under vigorous stirring in a $2 \mathrm{MHNO}_{3}$ solution. The precipitate was isolated again by magnetic decantation and dispersed in $15 \mathrm{ml}$ of a boiling solution containing $M\left(\mathrm{NO}_{3}\right)_{2}(0.15 M)$ and $\mathrm{Fe}\left(\mathrm{NO}_{3}\right)_{3}$. The precipitate obtained after this treatment was isolated, washed once with water and twice with acetone, and dispersed in $5 \mathrm{ml}$ of water by adding tetramethylammonium hydroxide until a stable ferrofluid was obtained. Residual acetone was removed in a moderate vacuum at $313 \mathrm{~K}$. The fluid was finally centrifuged at $4500 \mathrm{~g}$ for $0.35 \mathrm{~h}$ and the precipitate was discarded. The concentration of the nanoparticles was checked by thermogravimetric analysis and water was eventually added to the resulting ferrofluid so as to obtain a $5 \mathrm{wt} \%$ solid content.

\subsection{Hydrophobic modification}

An aliquot of the previously described ferrofluid $(2 \mathrm{ml}, 100 \mathrm{mg}$ of nanoparticles) was diluted with water to $50 \mathrm{ml} .5 \mathrm{ml}$ of a $2 \mathrm{wt} \%$ dodecanoic acid solution in acetone was then added to the diluted ferrofluid. The solution was heated to $353 \mathrm{~K}$ for $0.5 \mathrm{~h}$ under stirring to produce a water-based suspension, which was cooled to room temperature. Acetone and $\mathrm{MeOH}$ were added to precipitate the particles, which were then isolated by magnetic decantation and washed three times with acetone and $\mathrm{MeOH}$. The particles were dried and finally dispersed in deuterated cyclohexane to form a stable magnetic fluid.

\subsection{Small-angle scattering of polarized neutrons}

Small-angle scattering of polarized neutrons (SANSPOL) measurements were performed with the V4 instrument at the BERII reactor of the Hahn-Meitner Institute, Berlin (Keller et al., 2000). A neutron wavelength $\lambda$ of $6 \AA$ and detector distances of 1,4 and $12 \mathrm{~m}$ were used, corresponding to a $Q$ range $[Q=(4 \pi / \lambda) \sin (\theta / 2)$, where $\theta$ is the scattering angle] of $0.005-0.3 \AA^{-1}$. A horizontal magnetic field strength up to about $1 \mathrm{~T}$ was applied at the sample position, oriented perpendicularly to the incoming neutrons. Polarized neutrons are provided by a transmission polarizing supermirror cavity. The polarization direction is reversed using a spin flipper in front of the sample.

For polarized neutrons, where the neutron spins are aligned antiparallel $(+)$ or parallel $(-)$ to the magnetic field vector $\mathbf{H}$, the scattering cross sections depend on the polarization of the incident neutrons $I^{+}(Q)$ and $I^{-}(Q)$, respectively. The scattering intensity relationships (denoted here as SANSPOL intensities) have been previously derived (Wiedenmann, 2000, 2002). When the magnetic moments and neutron polarization are fully aligned along the external field, the SANSPOL intensities perpendicular to the applied field are given for the two states by
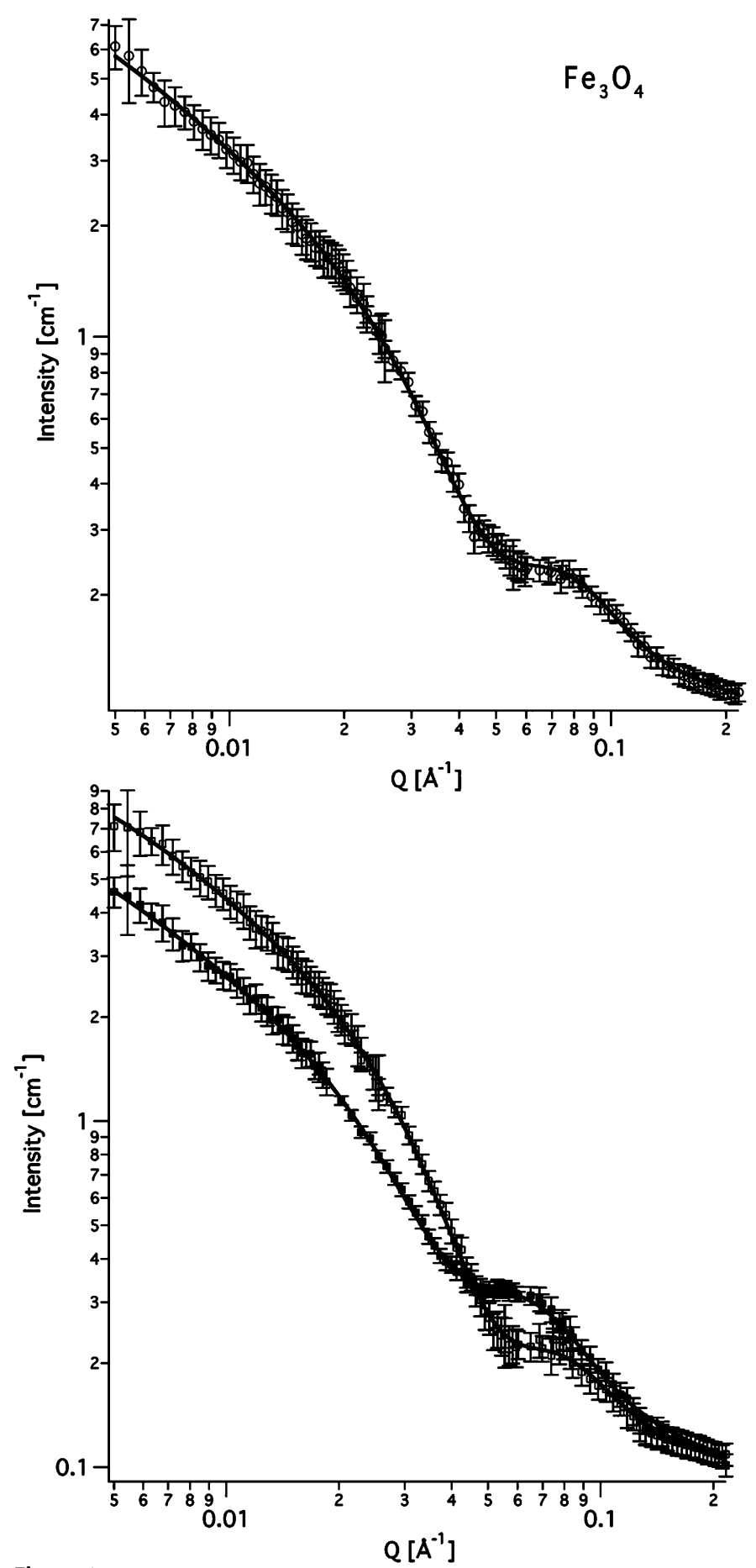

Figure 1

SANSPOL intensities parallel (top) and perpendicular [bottom, $I^{+}(Q \perp H)$ indicated by open squares and $I^{-}(Q \perp H)$ indicated by filled squares] to the applied field for $\mathrm{Fe}_{3} \mathrm{O}_{4}$ ferrofluid. Solid lines represent the best fitting results according to the pearl-necklace model. 
Table 1

Results obtained by fitting SANSPOL data to the pearl-necklace model.

\begin{tabular}{|c|c|c|c|}
\hline & $\mathrm{Fe}_{3} \mathrm{O}_{4}$ & $\mathrm{CoFe}_{2} \mathrm{O}_{4}$ & $\mathrm{CuFe}_{2} \mathrm{O}_{4}$ \\
\hline Volume fraction $\left(\times 10^{-2}\right)$ & $1.070 \pm 0.135$ & $0.753 \pm 0.036$ & $2.241 \pm 0.147$ \\
\hline Core radius $(\AA)$ & $38.9 \pm 1.9$ & $49.1 \pm 1.6$ & $39.1 \pm 4.3$ \\
\hline Core polydispersity, $\sigma_{\mathrm{c}}$ & $0.30 \pm 0.03$ & $0.32 \pm 0.02$ & $0.48 \pm 0.05$ \\
\hline Shell thickness $(\AA)$ & $7.6 \pm 1.3$ & $8.0 \pm 1.1$ & $6.7 \pm 2.1$ \\
\hline $\begin{array}{l}\text { SLD core, parallel } \\
\qquad\left(\times 10^{-6} \mathrm{~A}^{-2}\right)\end{array}$ & $6.933 \pm 0.009$ & $5.901 \mathrm{E}-6 \pm 0.008$ & $6.491 \pm 0.016$ \\
\hline $\begin{array}{l}\text { SLD core, perpendicular, } \\
\text { on }\left(\times 10^{-6} \AA^{-2}\right)\end{array}$ & $7.258 \pm 0.009$ & $6.253 \mathrm{E}-6 \pm 0.008$ & $6.630 \pm 0.017$ \\
\hline $\begin{array}{l}\text { SLD core, perpendicular, } \\
\text { off }\left(\times 10^{-6} \AA^{-2}\right)\end{array}$ & $6.606 \pm 0.009$ & $5.557 \mathrm{E}-6 \pm 0.008$ & $6.352 \pm 0.015$ \\
\hline SLD shell $\left(\times 10^{-6} \AA^{-2}\right)$ & $0.089 \pm 0.012$ & $0.089 \pm 0.018$ & $0.089 \pm 0.013$ \\
\hline Fractal dimension, $D$ 。 & $1.076 \pm 0.166$ & $1.621 \pm 0.285$ & $1.176 \pm 0.264$ \\
\hline Correlation length $(\AA)$ & $544.5 \pm 163.5$ & $192.3 \pm 73.6$ & $158.0 \pm 89.0$ \\
\hline
\end{tabular}

$$
I^{(-,+)}(Q \perp H) \propto\left[P_{\mathrm{N}} \pm P_{\mathrm{M}}\right]^{2} S(Q),
$$

where $P_{\mathrm{N}}$ and $P_{\mathrm{M}}$ represent the nuclear and magnetic form factors, respectively, and $S(Q)$ is the interparticle structure factor.

The arithmetic mean of the parallel and antiparallel intensities perpendicular to the applied field corresponds to the cross section for unpolarized neutrons:

$$
\left[I^{-}(Q \perp H)+I^{+}(Q \perp H)\right] / 2=I^{\mathrm{unpol}}(Q \perp H) \propto\left[P_{\mathrm{N}}^{2}+P_{\mathrm{M}}^{2}\right] S(Q) .
$$

The scattering cross section parallel to $\mathbf{H}$ is independent of the polarization state since it depends on pure nuclear contrast and is given by

$$
I(Q \| H) \propto P_{\mathrm{N}}^{2} S(Q) .
$$

The difference between the two intensities represents a magnetic nuclear cross term, allowing the magnetic contrast with respect to the nuclear contrast to be determined:

$$
I^{-}(Q, \alpha)-I^{+}(Q, \alpha) \propto P_{\mathrm{N}} P_{\mathrm{M}} S(Q),
$$

where $\alpha$ is the azimuth angle between the magnetic field vector $\mathbf{H}$ and the scattering vector $\mathbf{Q}\left(\mathbf{Q}=\mathbf{k}_{\mathrm{i}}-\mathbf{k}_{\mathrm{s}}\right.$, where $\mathbf{k}_{\mathrm{i}}$ and $\mathbf{k}_{\mathrm{s}}$ are the incident and scattering wavevectors, respectively).

Both the perpendicular and the parallel intensities have been calculated in two separate ways, obtaining identical results: (i) by adjusting the two-dimensional (2D) pattern to the $\sin ^{2} \alpha$ dependence, and (ii) by averaging the $2 \mathrm{D}$ pattern only over sectors with a width of $5^{\circ}$ and centred at $\alpha=90$ and $270^{\circ}$ for the perpendicular intensities, and centred at $\alpha=0$ and $180^{\circ}$ for the parallel intensities. As expected, the parallel intensities for the two flipper states were identical: in fact, as indicated in equation (4), these intensities account only for the nuclear scattering.

\section{Results and discussion}

Hydrophobic iron ferrite nanoparticles have been synthesized by grafting dodecanoic acid onto the surface of ferrite nanoparticles prepared through alkaline co-precipitation. These hydrophobically modified nanoparticles were dispersed in cyclohexane to form stable ferrofluids that were investigated by using the SANSPOL approach.

Scattering results were fitted according to the model introduced by Chen \& Teixeira (1986) for the analysis of the structure and the fractal dimension of protein-detergent complexes. This model was developed to describe the scattering pattern produced by a string of spherical micelles distributed along a polypeptide chain, nicknamed a 'pearl necklace'. Chen and Teixeira's pearl-necklace model neglects the contribution arising from the peptide chain. This approximation
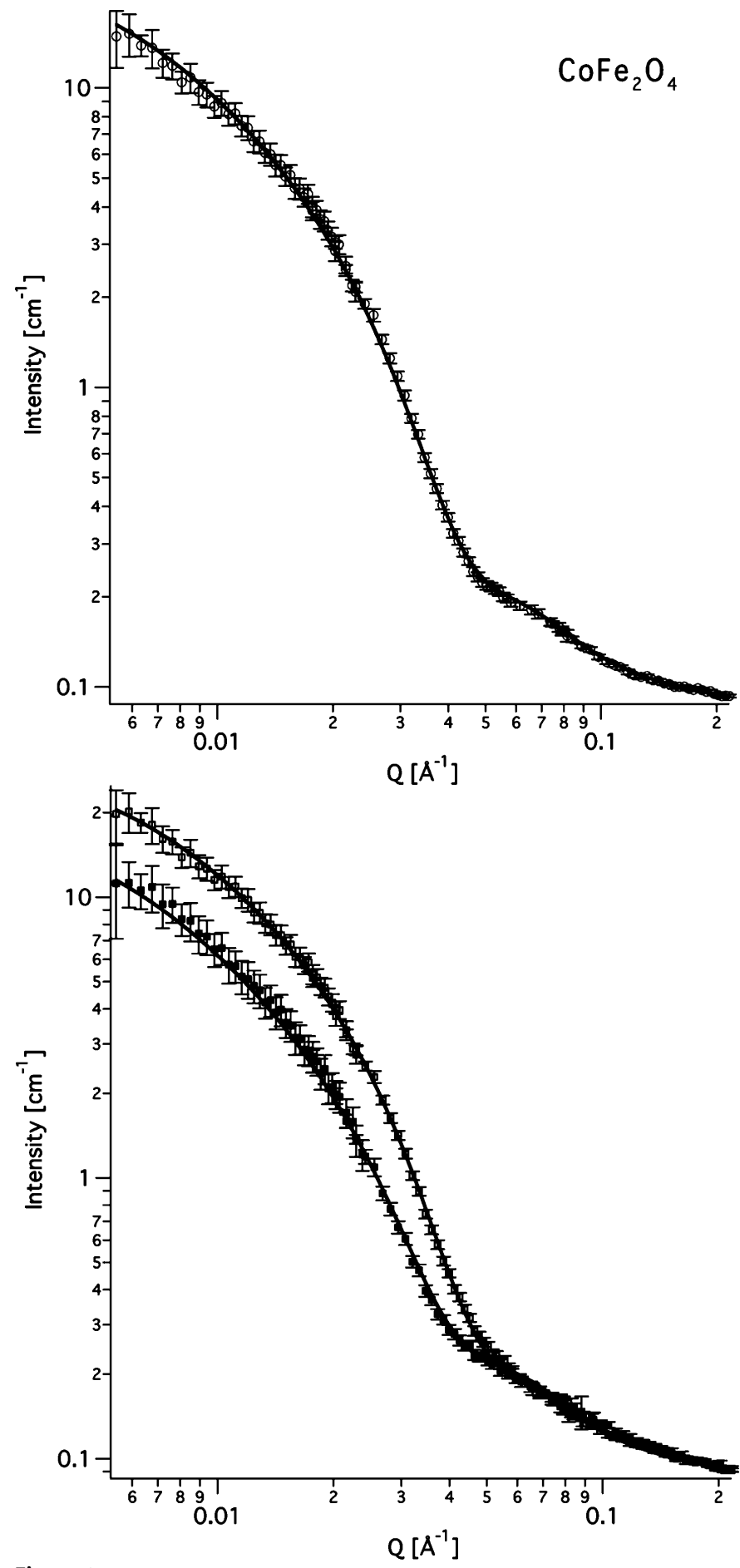

Figure 2

SANSPOL intensities parallel (top) and perpendicular [bottom, $I^{+}(Q \perp H)$ indicated by open squares and $I^{-}(Q \perp H)$ indicated by filled squares] to the applied field for $\mathrm{CoFe}_{2} \mathrm{O}_{4}$ ferrofluid. Solid lines represent the best fitting results according to the pearl-necklace model.

was justified by the fact that the major contrast was between the micelles and the solvent, i.e. the radiation was scattered only by the 'pearls' and not by the backbone. In our case the same approximation is legitimate because of the absence of a backbone, since the nanoparticles are eventually assembled in aggregates only because of their magnetic moment interactions. 

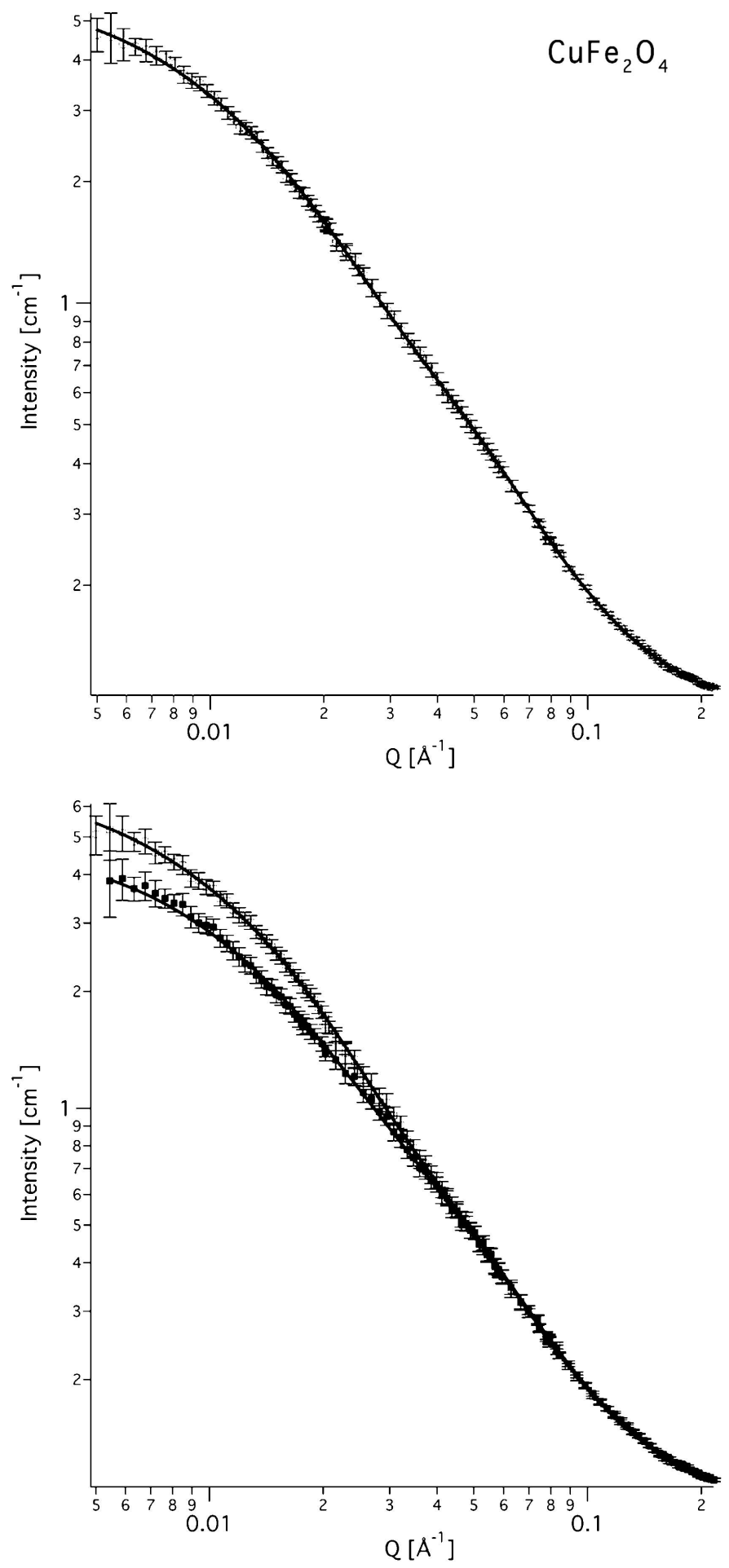

Figure 3

SANSPOL intensities parallel (top) and perpendicular [bottom, $I^{+}(Q \perp H)$ indicated by open squares and $I^{-}(Q \perp H)$ indicated by filled squares] to the applied field for $\mathrm{CuFe}_{2} \mathrm{O}_{4}$ ferrofluid. Solid lines represent the best fitting results according to the pearl-necklace model.

In a SANS experiment, the measured intensity of the radiation, $I(Q)$, scattered by a collection of uniform particles is given by (Guinier \& Fournet, 1955; Chen \& Teixeira, 1986)

$$
I(Q)=\phi P(Q) S(Q)+\mathrm{bkg}
$$

where $\phi$ is the particle volume fraction, $P(Q)$ is the form factor, $S(Q)$ is the interparticle structure factor accounting for the interparticle correlations, and bkg is the incoherent background.

Chen and Teixeira used the form factor $P(Q)$ of a sphere to describe the micelles. In this study we cannot consider a simple sphere as form factor since the magnetic particles comprise a metallic core and an organic shell. Therefore, we modified the model by introducing the particle form factor proposed by Bartlett and Ottewill for polydisperse spherical particles with a core-shell structure (Bartlett \& Ottewill, 1992). In this form factor the spherical particles have a constant shell thickness and a core with a Schulz distribution of radii. The contribution to the total scattering intensity arising from these objects was calculated according to

$$
\begin{gathered}
P(Q)=\left(1 / V_{\mathrm{p}}\right) \int_{0}^{\infty} G\left(r_{\mathrm{c}}\right) F^{2}\left(Q r_{\mathrm{c}}\right) \mathrm{d} r_{\mathrm{c}} \\
F\left(Q r_{\mathrm{c}}\right)=\left(4 \pi / Q^{3}\right)\left(\rho_{\text {shell }}-\rho_{\text {core }}\right)\left\{\rho_{\text {scaled }} j\left[Q r_{\mathrm{c}}+\left(t / r_{\mathrm{c}}\right) Q r_{\mathrm{c}}\right]-j\left(Q r_{\mathrm{c}}\right)\right\} \\
\rho_{\text {scaled }}=\left(\rho_{\text {solv }}-\rho_{\text {shell }}\right)\left(\rho_{\text {core }}-\rho_{\text {shell }}\right)
\end{gathered}
$$

and

$$
j\left(Q r_{\mathrm{c}}\right)=\sin \left(Q r_{\mathrm{c}}\right)-\left(Q r_{\mathrm{c}}\right) \cos \left(Q r_{\mathrm{c}}\right),
$$

where $r_{\mathrm{c}}$ is the core radius, $t$ is the shell thickness, $V_{\mathrm{p}}$ is the particle volume, and $\rho_{\text {core }}, \rho_{\text {shell }}$ and $\rho_{\text {solv }}$ are the scattering length densities (SLDs) of the core, shell and solvent, respectively.

The function $G\left(r_{\mathrm{c}}\right)$ is the normalized probability of finding a particle with a core radius between $r_{\mathrm{c}}$ and $r_{\mathrm{c}}+\mathrm{d} r_{\mathrm{c}}$, and it accounts for the polydispersity of the cores according to a Schultz distribution (Hayter \& Penfold, 1981; Hayter, 1983):

$$
G\left(r_{\mathrm{c}}\right)=\frac{r_{\mathrm{c}}^{Z}}{\Gamma(Z+1)}\left(\frac{Z+1}{\left\langle r_{\mathrm{c}}\right\rangle}\right)^{Z+1} \exp \left[-\frac{r_{\mathrm{c}}}{r_{\mathrm{avg}}}(Z+1)\right],
$$

where $\Gamma(Z+1)$ is the gamma function and the parameter $Z$ is related to the polydispersity $\sigma_{\mathrm{c}}$ of the core radius by the expression

$$
\sigma_{\mathrm{c}}=\frac{\left(\left\langle r_{\mathrm{c}}^{2}\right\rangle-\left\langle r_{\mathrm{c}}\right\rangle^{2}\right)^{1 / 2}}{\left\langle r_{\mathrm{c}}\right\rangle}=\frac{1}{(Z+1)^{1 / 2}} .
$$

The interparticle structure factor $S(Q)$ describes how the scattering intensity is modulated by interference effects between radiation scattered by different scattering objects. In our case, it should account for the aggregation of the magnetic nanoparticles, in analogy with the distribution of micelles along the polypeptide backbone. Therefore, we used the same expression derived by Chen and Teixeira,

$$
S(Q)=1+\frac{D \Gamma(D-1)}{(Q r)^{D}} \sin \left[(D-1) \tan ^{-1}(Q \xi)\right]\left(1+\frac{1}{Q^{2} \xi^{2}}\right)^{(1-D) / 2},
$$

where $r$ is the mean radius of the particles as resulting from the sum of the core radius $r_{\mathrm{c}}$ and the shell thickness $t, \Gamma$ is the gamma function and $\xi$ is the correlation length, i.e. a cut-off factor related to the dimensions of the aggregates that are eventually formed by the particles. $D$ is the fractal dimension that describes the spatial distribution of the individual scatterers and it is related to their number $N(R)$ within a sphere of radius $R$ through

$$
N(R)=(R / r)^{D}
$$

SANSPOL intensities of $\mathrm{Fe}_{3} \mathrm{O}_{4}, \mathrm{CoFe}_{2} \mathrm{O}_{4}$ and $\mathrm{CuFe}_{2} \mathrm{O}_{4}$ are shown in Figs. 1, 2 and 3, respectively. The curves resulting from the fitting 
obtained according to the modified pearl-necklace model are shown as full lines in the corresponding figures, while the parameters extracted from the analysis of the SANSPOL data are reported in Table 1 . The fitting routine has been constrained to globally fit all the SANSPOL curves for each sample, i.e. the fitting parameters are the same for the SANSPOL intensities parallel and perpendicular (flipper on and off) to the magnetic field, except for the core scattering length density that changes as a function of the polarization of the neutrons and the angle between $\mathbf{Q}$ and $\mathbf{H}$. This is summarized in equation (14), where the nuclear part of the core scattering length density is the same for all the SANSPOL intensities, while the magnetic contribution is null for the parallel direction and it has the same value for the two perpendicular curves:

$$
\rho_{\text {core }}=\rho_{\text {core }}^{\text {nucl }} \pm \rho_{\text {core }}^{\text {mag }} .
$$

Results of the fitting show that the nanoparticles comprise a magnetic core with a mean radius of about $40-50 \AA$ and a polydispersity ranging from 0.3 to 0.5 . In detail, the mean radii of the magnetic cores are very similar in the cases of $\mathrm{Fe}_{3} \mathrm{O}_{4}$ and $\mathrm{CuFe}_{2} \mathrm{O}_{4}$ (about $40 \AA$ ), while the value is slightly higher in the case of $\mathrm{CoFe}_{2} \mathrm{O}_{4}$ (about $50 \AA$ ). Moreover, the polydispersity value is very similar in the cases of $\mathrm{Fe}_{3} \mathrm{O}_{4}$ and $\mathrm{CoFe}_{2} \mathrm{O}_{4}$ (about 0.3) and higher for $\mathrm{CuFe}_{2} \mathrm{O}_{4}$ (close to 0.5). The shell thickness is similar in all the samples, ranging from 6.7 to $8.0 \AA$.

The SANSPOL analysis shows that fractal aggregates are present in all the samples, with different fractal dimensions and correlation lengths. In $\mathrm{Fe}_{3} \mathrm{O}_{4}$ and $\mathrm{CuFe}_{2} \mathrm{O}_{4}$ the fractal dimension is quite close to one, accounting for almost linear aggregates, while the fractal dimension is about 1.6 in $\mathrm{CoFe}_{2} \mathrm{O}_{4}$. The correlation length values range from about $160 \AA$ for the copper ferrite to $190 \AA$ for the cobalt ferrite and $550 \AA$ for the iron ferrite sample. It is important to note that the correlation length values are close to the higher limit of the instrumental size range. This is the main reason why the error values in their determination are quite high, indicating a low accuracy in the obtained correlation lengths.

\section{Conclusions}

This work reports the synthesis and the SANSPOL characterization of ferrofluids comprising ferrite nanoparticles coated by dodecanoic acid and dispersed in cyclohexane. Small-angle scattering of polarized neutrons takes advantage of the modification of the magnetic cross section and allows the determination of both magnetic and diamagnetic domains that could be present in nanostructured magnetic fluids. The results obtained from the pearl-necklace model show that the composition of the magnetic cores of the ferrite nanoparticles affects both the mean size of the particles and the particle size distribution. These, in turn, affect the formation of aggregates under an applied magnetic field, both in terms of fractal dimension and correlation lengths.

This project has been supported by Consorzio per lo sviluppo dei Sistemi a Grande Interfase (CSGI, Italy), Ministero per l'Istruzione, l'Università e la Ricerca (MIUR, PRIN-2006, Italy) and the European Commission under the 6th Framework Programme through the Key Action: Strengthening the European Research Area, Research Infrastructures, contract No. RII3-CT-2003-505925 (NMI3).

\section{References}

Bartlett, P. \& Ottewill, R. H. (1992). J. Chem. Phys. 96, 3306-3318.

Berbenni, V., Marini, A., Matteazzi, P., Ricceri, R. \& Welham, N. J. (2003). J. Eur. Ceram. Soc. 23, 527-536.

Berkovsky, B. \& Bashtovoy, V. (1996). Magnetic Fluids and Applications Handbook. New York: Begell House Inc.

Bonini, M., Bardi, U., Berti, D., Neto, C. \& Baglioni, P. (2002). J. Phys. Chem. $B, \mathbf{1 0 6}, 6178-6183$.

Bonini, M., Wiedenmann, A. \& Baglioni, P. (2004a). J. Phys. Chem. B, 108, 14901-14906.

Bonini, M., Wiedenmann, A. \& Baglioni, P. (2004b). Phys. A Stat. Mech. Appl. 339, 86-91.

Chen, S. H. \& Teixeira, J. (1986). Phys. Rev. Lett. 57, 2583-2586.

Gennes, P. G. de \& Pincus, P. A. (1970). Phys. Kondens. Mater. 11, 189-198.

Gross, M. \& Kiskamp, S. (1997). Phys. Rev. Lett. 79, 2566-2569.

Guinier, A. \& Fournet, G. (1955). Small Angle Scattering of X-rays. New York: John Wiley.

Hayter, J. (1983). Physics of Amphiphiles: Micelles, Vesicles and Microemulsions, edited by V. DeGiorgio \& M. Corti, pp. 59-93. Amsterdam: North Holland.

Hayter, J. B. \& Penfold, J. (1981). Mol. Phys. 42, 109-118.

Hyeon, T. (2003). Chem. Commun. pp. 927-934.

Hyeon, T., Lee, S. S., Park, J., Chung, Y. \& Bin Na, H. (2001). J. Am. Chem. Soc. 123, 12798-12801.

Jacobs, I. S. \& Bean, C. P. (1963). Magnetism, edited by G. T. Rado \& H. Suhl. New York: Academic Press.

Keller, T., Krist, T., Danzig, A., Keiderling, U., Mezei, F. \& Wiedenmann, A. (2000). Nucl. Instrum. Methods Phys. Res. A, 451, 474-479.

Lalatonne, Y., Richardi, J. \& Pileni, M. P. (2004). Nature Mater. 3, 121-125.

Leslie-Pelecky, D. L. \& Rieke, R. D. (1996). Chem. Mater. 8, 1770-1783.

Liu, J., Lawrence, M., Wu, A., Ivey, M. L., Flores, G. A., Javier, K., Bibette, J. \& Richard, J. (1995). Phys. Rev. Lett. 74, 2828-2831.

Massart, R. (1980). C. R. Acad. Sci. Ser. C, 291, 1-3.

Massart, R. (1981). IEEE Trans. Magn. 17, 1247-1248.

Massart, R. (1982). US Patent 4329241.

Papell, S. S. (1965). US Patent 3215572.

Park, J., Lee, E., Hwang, N. M., Kang, M. S., Kim, S. C., Hwang, Y., Park, J. G., Noh, H. J., Kini, J. Y., Park, J. H. \& Hyeon, T. (2005). Angew. Chem. Int. Ed. 44, 2872-2877.

Sun, S. H. \& Murray, C. B. (1999). J. Appl. Phys. 85, 4325-4330.

Toor, W. R. (1993). J. Colloid Interface Sci. 156, 335-349.

Wiedenmann, A. (2000). J. Appl. Cryst. 33, 428-432.

Wiedenmann, A. (2002). Lecture Notes in Physics, edited by S. Odenbach, pp. 33-61. Berlin: Springer.

Wiedenmann, A., Hoell, A. \& Kammel, M. (2002). J. Magn. Magn. Mater. 252, $83-85$. 